\title{
The effects of metallicity, radiation field and dust extinction on the charge state of PAHs in diffuse clouds: implications for the DIB carrier
}

\author{
N. L. J. Cox ${ }^{1}$ and M. Spaans ${ }^{2}$ \\ 1 Astronomical Institute "Anton Pannekoek", Universiteit van Amsterdam, Kruislaan 403, 1098-SJ Amsterdam, The Netherlands \\ e-mail: ncox@science.uva.nl \\ 2 Kapteyn Astronomical Institute, University of Groningen, PO Box 800, 9700-AV Groningen, The Netherlands
}

Received 7 November 2005 / Accepted 27 January 2006

\begin{abstract}
Context. The unidentified diffuse interstellar bands (DIB) are observed throughout the Galaxy, the Local Group and beyond. Their carriers are possibly related to complex carbonaceous gas-phase molecules, such as (cationic) polycyclic aromatic hydrocarbons and fullerenes.

Aims. In order to reveal the identity of the DIB carrier we investigate the effects of metallicity, radiation field and extinction curve on the PAH charge state distribution, and thus the theoretical emergent PAH spectrum, in diffuse interstellar clouds. This behaviour can then be linked to that of the DIB carrier, thus giving insight into its identity.

Methods. We use radiative transfer and chemical models to compute the physical and chemical conditions in diffuse clouds with Galactic and Magellanic Cloud types of interstellar dust and gas. Subsequently, the PAH charge state distributions throughout these clouds are determined.

Results. We find that the fraction of PAH cations is much higher in the Magellanic Cloud environments than in the Milky Way, caused predominantly by the respective lower metallicities, and mitigated by the steeper UV extinction curve. The fraction of anions is much lower in a low metallicity environment. The predicted DIB strength of cationic PAH carriers is similar to that of the Milk Way for the LMC and $40 \%$ for the SMC due to the overall metallicity. Stronger DIBs could be expected in the Magellanic Clouds if they emanate from clouds that are exposed to an average interstellar radiation field that is significantly stronger than in the Milky Way, although photo-destruction processes could possibly reduce this effect, especially for the smaller PAHs. Our results show that the presence and absence of DIB carriers in the Magellanic Cloud lines of sight can be tied to the PAH charge balance which is driven by metallicity, UV radiation and dust extinction effects.
\end{abstract}

Key words. astrochemistry - ISM: clouds - ISM: dust, extinction - ISM: molecules - ISM: lines and bands - Magellanic Clouds

\section{Introduction}

In the last decade complex carbonaceous molecules (e.g. carbon chains, polycyclic aromatic hydrocarbons and fullerenes) have become viable candidates for the carriers of the numerous, as of yet unidentified, diffuse interstellar bands (DIBs). Observations of infrared characteristics of C-H and C-C stretching and bending modes in PAHs have added to the believe in this hypothesis. Since their discovery (Heger 1922) many carriers have been put forward, but none have been confirmed (see e.g. Herbig 1995 for a review).

Early observations of extra-galactic DIBs had to focus on the very broad, shallow, but strong $4430 \AA$ DIB, but recently it has become feasible to observe the strongest narrow DIBs in Magellanic Cloud lines of sight (e.g. Ehrenfreund et al. 2002) and even beyond toward supernovae in nearby galaxies (Sollerman et al. 2005). This allows for a study of the effects of, for example, metallicity on the DIB carrier formation and destruction processes. Detailed analyses of the DIBs and the interstellar medium (ISM) of our nearest neighbours, the Large and Small Magellanic Clouds, show DIB behaviour that is similar to the MW in some sightlines while different in others (Cox et al. 2006a,b; Welty et al. 2006).

To understand the life cycle and evolution of the DIB carrier(s) we need to grasp some of the complexity of the chemistry and physics of the ISM. Unfortunately, there are still many caveats in our understanding of the chemistry in the diffuse interstellar medium, both in the Milky Way and beyond. The effect of one of the fundamental differences between galaxies in the Local Group, i.e. their metallicity, warrants further examination. Metallicities have been discussed for photo-dissociation region (PDR) models, see e.g. Maloney \& Black (1988), Van Dishoeck \& Black (1988), Wolfire et al. (1989), Maloney \& Wolfire (1996), and for a more specific application to the LMC, see e.g. Pak et al. (1998), Kaufman et al. (1999), Stutzki (2001), Jeyakumar \& Stutzki (2003). From these studies it has become clear that the metallicity affects the local physical and chemical conditions. To apply the cloud chemistry models designed for Milky Way lines of sight to other galaxies we need to take into account several fundamental differences in the nature/composition of the ISM in these external galaxies. Besides the decrease in metallicity (and related to that the decrease in the dust-to-gas ratio), these galaxies also differ in the gas and dust 
composition, i.e. the extinction curve, as well as the interstellar background UV radiation field.

The relative abundance deficiencies, compared to the local ISM, are $\sim-0.3$ dex (Rolleston et al. 2002, and references therein) and $\sim-0.6$ dex (Lee et al. 2005 and references therein) for the LMC and SMC, respectively. The (dust) extinction properties differ between the MW, LMC and SMC. For example, on average the LMC and SMC gas-to-dust ratios are about 4 and 10 times higher than in the MW, respectively (e.g. Bohlin et al. 1978; Gordon et al. 2003; Cartledge et al. 2005). Recent results by Misselt et al. (1999) and Gordon et al. (2003) showed that lines of sight toward 30 Doradus have a rather steep FUV rise, and a weak $2175 \AA$ bump (often related to carbonaceous material), whereas the rest of the LMC has extinction curves much more like those observed in our Galaxy. However, only four SMC extinction curves have been constructed thus far, of which one (AzV 456) does show a bump and a weak FUV rise (Rocca-Volmerange et al. 1981; Prevot et al. 1984; Gordon et al. 2003; Cartledge et al. 2005), and includes DIBs.

Ruiterkamp et al. (2005) investigated the PAH-DIB hypothesis (Leger \& D'Hendecourt 1985; Van der Zwet \& Allamandola 1985) by deriving how the charge state distribution for different (large) complex carbonaceous molecules (i.e. PAHs in this study) is determined by the local diffuse interstellar cloud conditions, such as e.g. the temperature (structure), the electron and hydrogen density, the UV field and the optical extinction within a diffuse cloud (similar to the treatment of PDRs by Bakes \& Tielens 1998). They compared the resulting theoretical PAH absorption spectrum to the observed DIB spectrum for the translucent line of sight toward HD 147889, and showed that the strong DIBs, if indeed PAHs, are probably related to specific series of PAHs that have strong cationic electronic transitions.

The aim of this paper is to quantify the effect of metallicity, (dust) extinction properties and the interstellar radiation field on the cloud chemistry in general, and the PAH charge state in particular. In Sect. 2 we outline our previously constructed diffuse cloud model (Ruiterkamp et al. 2005), and the incorporation of the metallicity/elemental abundances. The next section describes first the results for clouds with Galactic type dust but different metallicities (Sect. 3.1) and for the low metallicity ISM in Magellanic Cloud type environments (Sect. 3.2). Section 4 shows how these different environments affect the emerging diffuse cloud PAH absorption spectrum. We discuss the consequences for the PAH-DIB carrier hypothesis in Sect. 5, and conclude with an application of the results to two well studied SMC sightlines that probe two very different interstellar diffuse cloud environments.

\section{The diffuse cloud model}

\subsection{Chemical and thermal structure and the PAH charge state distribution}

The PAH charge state distribution is determined by the reaction rates for photo-ionization, electron recombination, electron attachment and photo-detachment. These reactions depend on the local interstellar conditions (e.g. temperature $T$, electron density $n_{\mathrm{e}}$, hydrogen density $n_{\mathrm{H}}$ and effective strength of the interstellar radiation field $I_{\mathrm{UV}}$ ), as well as the properties of the considered molecule, e.g. the size (the number of carbon atoms $N_{\mathrm{C}}$ ) and the ionization potential (IP). We refer to Ruiterkamp et al. (2005) for the details on the adopted reaction rates. The basic properties (i.e. $N_{\mathrm{C}}$ and IP) of the selected PAHs (one kind of large carbonaceous molecules) are listed in Table 1.
Table 1. Basic properties of the selected PAHs: ionization potential IP $(\mathrm{eV})$ and number of carbon atoms $N_{\mathrm{C}}$. The two adopted types (1) and (2) correspond to two PAH symmetry groups (i.e. geometric structure) $\left(\mathrm{D}_{6 h}\right.$ coronenes and $\mathrm{D}_{2 h}$ acenes) that have a different IP- $N_{\mathrm{C}}$ dependence. Type (2) PAHs are more easily ionized than type (1). See also Fig. B.1. in Ruiterkamp et al. (2005). We assume a generic electron affinity of $3 \mathrm{eV}$, and a sticking coefficient of unity.

\begin{tabular}{|c|c|c|c|c|}
\hline \multirow[t]{2}{*}{$\overline{\#}$} & \multirow[t]{2}{*}{ 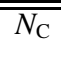 } & \multicolumn{2}{|c|}{$\overline{\overline{\mathrm{IP}}(\mathrm{eV})}$} & \\
\hline & & (1) & (2) & \\
\hline 1 & 20 & 7.6 & 6.8 & \\
\hline 2 & 30 & 7.2 & 6.2 & small \\
\hline 3 & 40 & 6.9 & 5.75 & \\
\hline 4 & 50 & 6.75 & 5.5 & loro \\
\hline 5 & 60 & 6.6 & 5.25 & large \\
\hline 6 & 80 & 6.4 & 4.9 & \\
\hline 7 & 100 & 6.3 & 4.7 & very large \\
\hline
\end{tabular}

Models for the chemical and thermal balance of the considered diffuse clouds (single-sided irradiated slabs) were constructed using the Monte Carlo code as described in Spaans (1996) and Spaans \& Van Dishoeck (1997). This code solves the gas phase chemical network and is driven by ion-molecule reactions, typical for diffuse clouds. Although the code does not include grain surface chemistry, dust is included as it causes the attenuation of the interstellar radiation field. Subsequently, the PAH charge state distribution is computed as a function of depth into the cloud.

\subsection{Metallicity and diffuse cloud chemistry}

Carbon and oxygen are important elements in the chemical network. Ionized carbon and carbon monoxide are the most critical species in the cooling, while the dust abundance is critical in heating the interstellar gas (i.e. lowering the dust grain abundance reduces the heating rate). Generic PAHs are also included in the chemical network and the thermal balance, with an abundance $n_{\mathrm{PAH}} / n_{\mathrm{H}}=10^{-7}$ for Solar metallicity (Bakes \& Tielens 1998). A lower $\mathrm{C}$ and $\mathrm{O}$ abundance makes it harder to sustain cool phase gas. This also happens when the UV radiation field is high (Wolfire et al. 1995). A lower dust abundance, and hence lower $\mathrm{H}_{2}$ formation rate and higher UV field intensity may cause $\mathrm{HI}$ to dominate over $\mathrm{H}_{2}$ even in regions with higher density (compared to the MW). This indicates that in the Magellanic Clouds H I absorption traces not only diffuse, but also denser clouds. These dense clouds remain atomic in the Magellanic Clouds, but would be molecular in the Milky Way (Dickey \& Garwood 1989).

The electron density is approximately related to the metallicity and the dust-to-gas ratio, via:

$n_{\mathrm{e}} \simeq n_{c^{+}} \propto \frac{Z}{Z_{\odot}} \propto \frac{A_{\mathrm{V}}}{N(\mathrm{HI})}$

with the dust-to-gas ratio scaling as $Z / Z_{\odot}$. Empirical evidence for this relationship for several galaxies of different metallicity was found by Issa et al. (1990). The gas-to-dust ratio will only affect the physical scale of the diffuse clouds, but not the extinction dependent cloud structure. The strength of the impinging UV field and the hydrogen density do affect the electron density and temperature structures. For example, for a cloud, with SMC extinction and metallicity, of physical depth $L=9 \times$ $10^{19} \mathrm{~cm}$ the cloud type B model $\left(I_{\mathrm{UV}} / n_{\mathrm{H}}=0.1\right.$, see Table 2$)$ gives $A_{\mathrm{V}}=0.5 \mathrm{mag}, n_{\mathrm{e}}=2.45 \times 10^{-5}$ and $T=203 \mathrm{~K}$, and for 
Table 2. Diffuse cloud physical parameters for the four distinct cloud configurations A, B, C and D. Each of these configurations consists of a single-sided irradiated slab with specified extinction properties and metallicities (for detailed discussion see main text, Sects. 3.1 and 3.2).

\begin{tabular}{lllll}
\hline \hline Cloud parameters & $A$ & $B$ & $C$ & $D$ \\
\hline$n_{\mathrm{H}}\left(\mathrm{cm}^{-3}\right)$ & 100 & 100 & 300 & 300 \\
$I_{\mathrm{UV}}$ & 1 & 10 & 1 & 10 \\
\hline
\end{tabular}

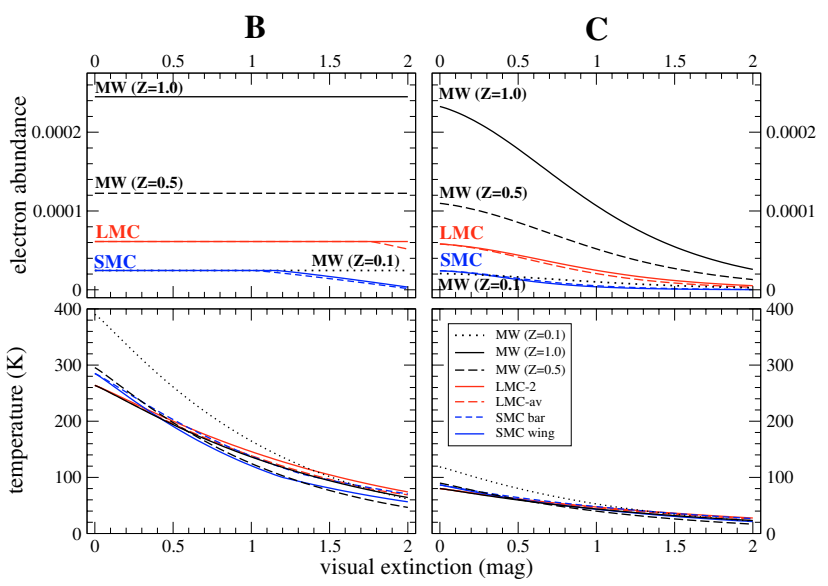

Fig. 1. The top left and right panel show the electron abundance structure for the diffuse cloud types $\mathrm{B}$ and $\mathrm{C}$, respectively. The respective basic cloud parameters are listed in Table 2. For each cloud type we show the results for different extinction curves and metallicities (Sects. 3.1 and 3.2). The bottom panels show the corresponding temperature structures. The local temperature and electron density are important for the charge state balance, and their extinction dependency is crucial to solve the PAH charge state throughout the diffuse cloud. Note the difference in electron density and temperature structure (as a function of extinction) between the two models B and C.

model $C\left(I_{\mathrm{UV}} / n_{\mathrm{H}}=0.0033\right.$; Table 2$) A_{\mathrm{V}}=1.5 \mathrm{mag}, n_{\mathrm{e}}=1.53 \times$ $10^{-6}$ and $T=36 \mathrm{~K}$, respectively.

Stutzki (2001) concluded that $N$ (dust) $\propto N_{\mathrm{C}} \propto Z$, and thus the same $A_{\mathrm{V}}$ is reached at a larger $\mathrm{H}_{2}$ column. The $I_{\mathrm{UV}} / n_{\mathrm{e}}$ ratio drives the charge balance. The shape of $I_{\mathrm{UV}}\left(A_{\mathrm{V}}\right)$ is independent of the dust-to-gas ratio, but $n_{\mathrm{e}}, f_{\mathrm{H}_{2}}\left(=\mathrm{H}_{2} / n_{\mathrm{H}}\right)$ and $T$ are a function of $Z / Z_{\odot}$ (for a given $A_{\mathrm{V}}$ ), the unattenuated interstellar radiation field and the observed extinction curve. Jeyakumar \& Stutzki (2003) conclude that for their PDR model (spherical cloud, $n_{\mathrm{H}}=10^{3} \mathrm{~cm}^{-3}$ ) the surface temperature is affected by $Z$ only for strong UV fields, $\log I_{\mathrm{UV}}>1$, whereas for weak fields, $\log I_{\mathrm{UV}}<1$, no change is predicted. This is consistent with our models that show a variation of the surface temperature and temperature structure with metallicity for UV field strengths of $I_{\mathrm{UV}}=1$ and 10 , with $n_{\mathrm{H}}=100$ and $300 \mathrm{~cm}^{-3}$ (Table 2). In terms of $\frac{I_{\mathrm{UV}}}{n_{\mathrm{H}}}$ both cases are equivalent.

The charge state of PAHs is (roughly) proportionally to $\frac{I_{\mathrm{UV}} \sqrt{T}}{n_{\mathrm{e}}}(\equiv \alpha)$, which depends on the availability of electrons for recombination, and is extinction dependent. See also Sect. 3 and Fig. 2. The main source of electrons, for strong UV fields, is the ionization of neutral carbon. At the cloud surface all the neutral carbon is ionized, therefore, $n_{\mathrm{e}} \sim n_{\mathrm{C}^{+}} \propto n_{\mathrm{C}^{+}, Z=1} Z$. Kaufman et al. (1999) show that for a weak radiation field (i.e. $I_{\mathrm{UV}} / n \lesssim 10^{-3}$ ) the $\mathrm{C}^{+}$is destroyed by collisions with neutral and anion PAHs. The abundance of neutral and anion PAHs increases with $C$ (and thus $Z$ ) because there are more gas phase electrons. Therefore, the $\mathrm{C}^{+}$abundance per $Z$ increases if $Z$ decreases.

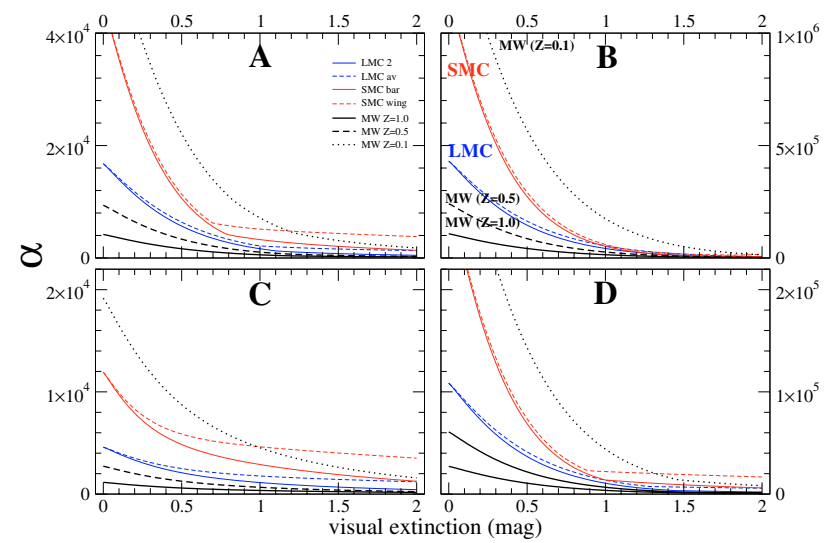

Fig. 2. $\alpha \equiv I_{\mathrm{UV}} \sqrt{T} /\left(x_{\mathrm{e}} n_{\mathrm{H}}\right)$ as a function of visual extinction $A_{\mathrm{V}}$, for the four cloud types and the Galactic and Magellanic Cloud metallicities and extinction curves (Sect. 3). $\alpha$ is roughly proportional to the PAH charge state (Sect. 2). $\alpha\left(A_{\mathrm{V}}\right)$ is shown for photons with a specific wavelength of $1600 \AA$ which corresponds to an energy of $7.8 \mathrm{eV}$. Note the different $y$-scales for each of the four panels. The ionization efficiency $\alpha$ increases strongly with an increase in $I_{\mathrm{UV}}$ as well as a decrease in metallicity $Z$, via the decrease in electron density.

\section{Applications}

\subsection{Milky way}

First we construct a small grid of diffuse clouds (a single-sided irradiated slab model, Spaans 1996) with typical Milky Way properties. We fix $n_{\mathrm{H}}=100$ or $300 \mathrm{~cm}^{-3}, I_{\mathrm{UV}}=1.0$ or 10.0 (see Table 2). The visual extinction $A_{\mathrm{V}}$, from the illuminated side of the slab to the dark side of the slab is set to 2.0 mag. We construct the models for the typical Galactic extinction curve, parametrized by $R_{\mathrm{V}}=3.1$ (Fig. 3, adopted from Fitzpatrick 1999, 2004) for a range in metallicity, $Z=1.0,0.5$ and $0.1 Z_{\odot}$. Figure 1 shows the electron density (top panels) and temperature (bottom panels) structure within the two cloud types B and C, for the different metallicities $Z$ with Galactic type (dust) extinction. The electron and density structure for cloud types $\mathrm{A}$ and $\mathrm{D}$ are not shown, since they are quite similar to cloud types B and C, respectively. In addition, Fig. 2 shows the extinction dependent charge balance indicator $\alpha$ (Sect. 2) for all four cloud types. These results for the diffuse cloud physical conditions are subsequently used to calculate the $A_{\mathrm{V}}$ dependent PAH charge state distribution. The charge state fractions of cations, neutrals and anions, as a function of $A_{\mathrm{V}}$, for the Galactic $\mathrm{B}$ and $\mathrm{C}$ cloud types (A and $\mathrm{D}$ omitted, but again very similar) are shown in Fig. 4. The effect of a decrease in metallicity on the total integrated charge state distribution is discussed in detail in Sect. 4.

\subsection{Magellanic clouds}

Now we apply the above results on the metallicity effects to diffuse clouds in the Large and Small Magellanic Clouds. However, before this can be done, there are two main points that need to be taken into account. First, we need to adopt a value for the overall metallicity and the gas phase metal abundance of the interstellar medium (ISM) in these Galaxies. We adopt $Z=0.25 Z_{\odot}$ and $0.10 Z_{\odot}$ for the LMC and SMC, respectively. Second, the model calculations will be affected not only by the metallicity but also by changes in the gas and dust composition. The appropriate extinction curves $\left(A_{\lambda} / A_{\mathrm{V}}\right)$ for the LMC (LMC-2 and LMC-av) and SMC (SMC bar and SMC wing) are those derived by Gordon et al. (2003) and further extended by Cartledge et al. (2005). 


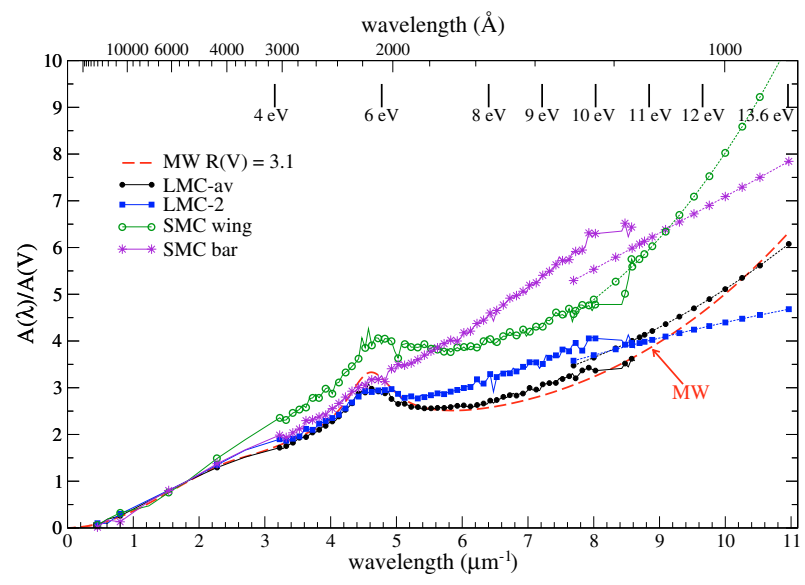

Fig. 3. Extinction curves adopted for the MW, LMC and SMC environments. Galactic extinction curve (dashed) adopted from Fitzpatrick (1999, 2004). Magellanic Cloud extinction curves (solid) adapted from Gordon et al. (2003) and Cartledge et al. (2005).

The adopted extinction curves are shown in Fig. 3. For each of these Magellanic Cloud extinction properties we calculate the charge state distribution for each of the four cloud types defined in Table 2. The extinction dependent ionization distribution for four specific PAHs (Table 1) is shown in Fig. 4 for the scenarios B and C, for each of the four Magellanic Cloud extinction and metallicity properties.

\section{Results}

How do the radiation field, the metallicity and the (dust) extinction properties affect the PAH charge balance, and subsequently the PAH absorption spectrum? In order to compare the derived model results with observations, the charge state distributions need to be averaged over the line of sight. The total (integrated) fractions of anions, neutrals and cations for the different scenarios and environments (MW, LMC and SMC) are shown in Fig. 5. Next we discuss the effect of the different environmental model parameters on the charge balance.

\subsection{The radiation field}

Increasing the strength of the radiation field $I_{\mathrm{UV}}$ increases the PAH ionization rate. For the investigated scenarios for diffuse clouds in the Galaxy and the Magellanic Clouds the results from Sect. 3.2 show that a tenfold increase of $I_{\mathrm{UV}}$ greatly enhances the fraction of cations and prohibits the presence of anions (fraction less than $0.5 \%)$. For thin diffuse clouds $\left(A_{\mathrm{V}}=1.0 \mathrm{mag}\right)$ the PAH cation fraction is larger than 90 and $80 \%$ for scenarios $B$ $\left(n_{\mathrm{H}}=100, I_{\mathrm{UV}}=10\right)$ and $D\left(n_{\mathrm{H}}=300, I_{\mathrm{UV}}=10\right)$, respectively. For slightly thicker clouds $\left(A_{\mathrm{V}}=2.0 \mathrm{mag}\right)$ the fraction of neutral PAHs (still no anions) increases up to 20 and $40 \%$ for scenarios $B$ and $D$, respectively. However, for larger PAHs the neutral fraction decreases again. For scenarios $A\left(n_{\mathrm{H}}=100\right.$, $\left.I_{\mathrm{UV}}=1\right)$ and $C\left(n_{\mathrm{H}}=300, I_{\mathrm{UV}}=1\right)$ the differences are more pronounced. Therefore, in the remainder of this section we will focus on the results for these scenarios which are summarized in Fig. 5. Note that all high energy UV photons are assumed to ionize PAHs, as photo-dissociation processes are not included. For diffuse clouds exposed to a strong $\left(I_{\mathrm{UV}}=10\right)$ radiation field the high energy photon flux could potentially lead also to the destruction of, mostly small, PAHs (see also Sect. 5).

\subsection{The metallicity}

For the Galactic diffuse clouds (Fig. 5), scenarios $A$ and $C$ show that a decrease in metallicity causes an increase in the cation fraction. This results directly from Eq. (1) which constitutes a proportionality between metallicity and the electron density. A lower electron density will result, all other parameters being equal, in a lower recombination rate, a lower electron attachment rate and thus a higher ionization fraction.

Let us consider the charge state fraction for a PAH with 20 carbon atoms and an ionization potential of $7.6 \mathrm{eV}$ (i.e. PAH \#1, Table 1). For scenario $C$ we have a relatively dense model cloud $\left(n_{\mathrm{H}}=300 \mathrm{~cm}^{-2}\right)$ which is irradiated by a relatively weak UV field $\left(I_{\mathrm{UV}}=1\right)$. The radiation is attenuated throughout the cloud $\left(A_{\mathrm{V}}=2.0 \mathrm{mag}\right)$ by Galactic type dust. For a gas with solar abundance the PAH cations, neutrals and anions constitute 2, 47 and 51\% (3, 42 and 55\%, respectively halfway through the cloud) of the total PAH column density. For regions with a lower metallicity (e.g. $0.1 Z_{\odot}$ ) the anion fraction decreases to $7 \%$, while the neutral and cation fractions both increase to 68 and $25 \%$. For larger PAHs $\left(N_{C}>40\right)$ the neutral fraction first increases with decreasing $Z$, but then decreases again as the cation fraction increases strongly, more than linearly, with decreasing $Z$. Due to the low metallicities, the Magellanic Cloud diffuse clouds show low anion and large cation fractions, similar to the low $Z$ Galactic clouds. The effect of dust extinction properties is discussed next.

\subsection{The extinction curve}

Introducing Magellanic Cloud dust extinction properties into the lower metallicity diffuse cloud models $\left(Z=0.25\right.$ and $\left.0.10 Z_{\odot}\right)$ changes the distribution of photons penetrating the cloud and thus changes the ionization balance. The results are shown in Fig. 5. Differences arise in charge state fractions between the MW $Z=0.1 Z_{\odot}$, SMC-wing and SMC bar cases, which differ in the adopted extinction curve. The small anion contributions $(0$ to $7 \%$ ) differ less than $3 \%$. The neutral fraction for both SMC extinction curves differs between 2 and 14\%, and the cation fraction between 3 to $14 \%$. Compared to the MW the SMC differ even more; the cation and neutral fractions are up to $15 \%$ higher and lower, respectively. There is little difference between the two LMC extinction curves, i.e. 1 to $6 \%$ differences. The more efficient attenuation of the far UV flux by the SMC dust (both bar and wing) results in a lower ionization fraction than that expected solely from the lower metallicity. Although the SMC bar and wing extinction curves differ in shape the overall ionizing radiation penetrating the dust results in a similar level of ionization. On the other hand, the bar region is exposed to relatively more high energy $(>11 \mathrm{eV})$ photons which are more prone to photo-dissociate PAHs (Sect. 5). On the other hand, these high energy photons are much more attenuated by the dust in the SMC than in the LMC or MW. Thus, for similar $I_{\mathrm{UV}}$ and $A_{\mathrm{V}}$ the PAHs are more protected against ionization and destruction in environments with SMC-like dust extinction properties (i.e. extinction curves). The electron density is directly related to the metallicity (Sect. 2.2), and the electron recombination rate is lower for lower metallicity environments, which results therefore in a higher ionization fraction.

\subsection{Diffuse versus translucent}

From the above it is already clear that the differences between $n_{\mathrm{H}}=100$ and $300 \mathrm{~cm}^{-3}$ are subtle, the latter showing an 

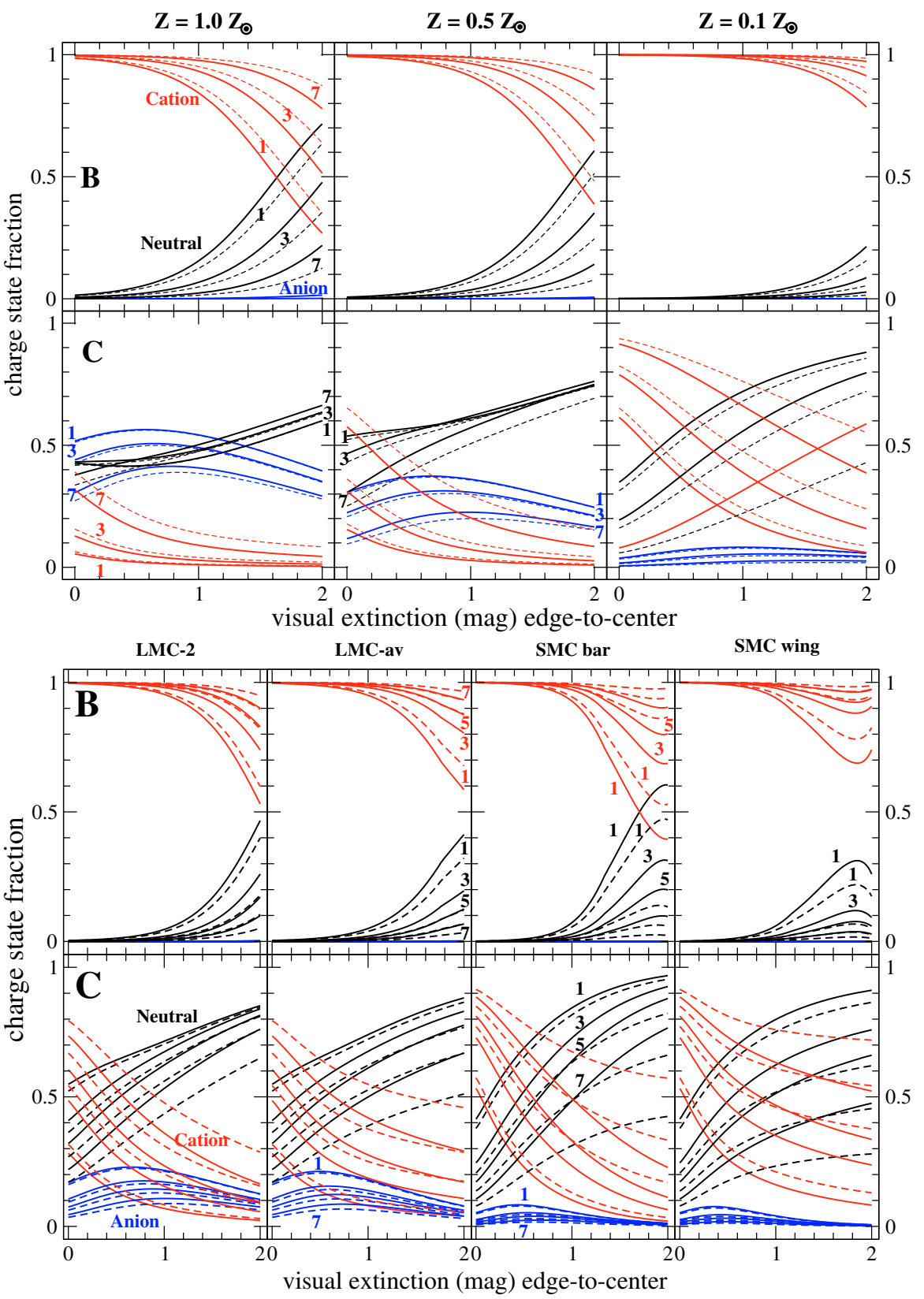

Fig. 4. Charge state distribution of PAHs as function of visual extinction $A_{\mathrm{V}}$. Each panel shows the cation (red), neutral (black) and anion (blue) fraction for the PAHs \#1, \#3 and \#7 of both type 1 (solid; $\mathrm{D}_{6 h}$ ) and 2 (dashed; $\left.\mathrm{D}_{2 h}\right)$ as listed in Table 1 . The scenar$\operatorname{ios} B\left(n_{\mathrm{H}}=100, I_{\mathrm{UV}}=10\right)$ and $C\left(n_{\mathrm{H}}=\right.$ $\left.300, I_{\mathrm{UV}}=1\right)$ are shown in the top and bottom panels, respectively. These two scenarios correspond to the two cloud types with the highest and lowest value for $I_{\mathrm{UV}} / n$. a) shows the results for Galactic $\left(R_{\mathrm{V}}=3.1\right)$ diffuse clouds. For each scenario the charge state fraction is given for three different metallicities: $Z=1.0,0.5$ and $0.1 Z_{\odot}$. A decrease in $Z$ shows an increase in the PAH cation fraction for both cloud models $B$ and $C$. For model $C$ the neutral and cation fractions are more sensitive to the $\mathrm{PAH}$ size for lower $Z$. This behaviour is not seen for model $B$. b) shows the PAH charge state versus $A_{\mathrm{V}}$ for Magellanic diffuse clouds. For each scenario the charge state fraction is given for the four different Magellanic Cloud dust extinction properties (LMC-2, LMC-av, SMC bar and SMC wing; see Fig. 3) and metallicities $\left(Z_{\mathrm{LMC}}=0.25 Z_{\odot}\right.$ and $Z_{\mathrm{SMC}}=$ $\left.0.10 Z_{\odot}\right)$. This figure shows the effect of the dust extinction properties (via the extinction curve) for both the LMC and SMC metallicities. Model $C$ shows the distinct difference in anion fraction between the LMC and SMC. overall larger fraction of anions and neutrals. The difference between "little" extinction $\left(A_{\mathrm{V}}=1.0 \mathrm{mag}\right.$, diffuse cloud) and "high" extinction ( $A_{\mathrm{V}}=2.0 \mathrm{mag}$, translucent cloud) clouds is similar, in that the total fraction of neutrals increases for higher $A_{\mathrm{V}}$ due to a higher fraction of neutrals towards the dark side of the slab. In practice, the observed (in the optical spectral range) diffuse cloud lines of sight in the LMC and SMC have visual extinctions less than one magnitude.

\section{Discussion: implications for the PAH-DIB carrier}

If indeed the DIB carriers are complex carbonaceous molecules, and more specifically, fullerenes or polycyclic aromatic hydrocarbons (PAHs) the DIB band strength should vary with their abundance along the line of sight. If furthermore the DIB carriers are PAHs or fullerenes in a certain charge state (e.g. anion, neutral or cation) then their abundance would vary with local interstellar conditions along the observed line of sight, due to shifts in the charge state balance. The total integrated PAH column density along the line of sight then directly yields the corresponding DIB strength. In practise, most lines of sight probe multiple diffuse clouds, each of which can have different environmental conditions that result in both a different total PAH column density and a specific PAH charge state balance. Nevertheless, several conclusions can be extracted from the results described above.

The total charge state fractions imply that the fraction of PAH cations is much higher in the Magellanic Cloud environment (due to the low metallicity) than in the Milky Way (solar metallicity). The dust extinction properties specific for the LMC and SMC, i.e. increased attenuation of high energy photons, counter the effect of lower metallicity and thus (slightly) mitigate the ionization level. The fraction of anions is much lower in the low metallicity environment. The carbon abundance, with respect to solar, is on average a factor 4 and 10 lower for the LMC and SMC, respectively. Thus, although the cation fraction for, for example, PAH \#7 $\left(N_{\mathrm{C}}=100, \mathrm{IP}=5 \mathrm{eV}\right)$ increases from 20 to $60 \%$ between MW and LMC, the PAH cation column 


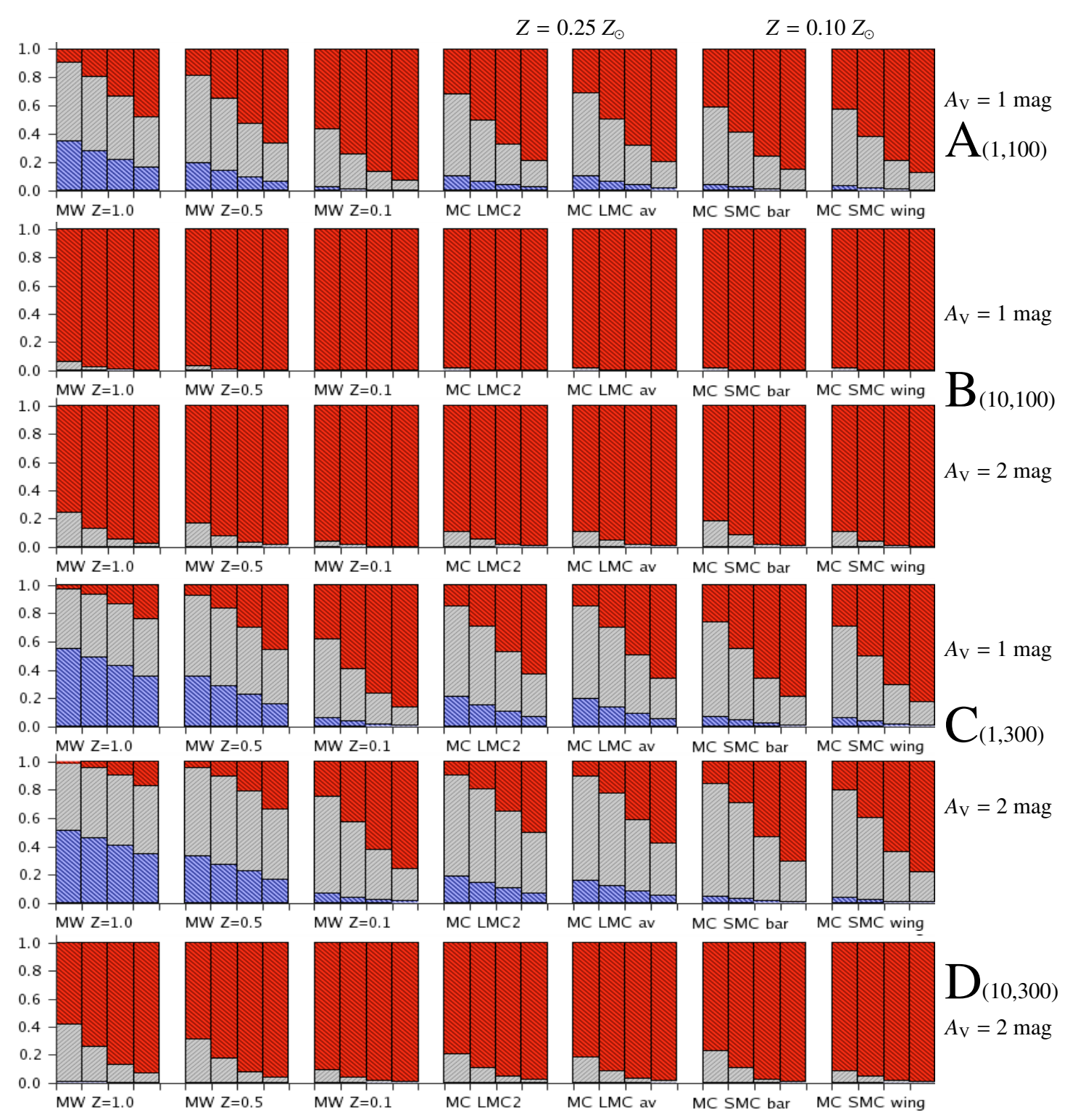

Fig. 5. Total (fractional) charge state distribution for cloud types $X\left(I_{\mathrm{UV}}, n_{\mathrm{H}}\right): A(1,100)\left(A_{\mathrm{V}}=1 \mathrm{mag}\right), B(10,100)\left(A_{\mathrm{V}}=1 \mathrm{and} 2 \mathrm{mag}\right), C(1,300)$ $\left(A_{\mathrm{V}}=1\right.$ and $2 \mathrm{mag}$ ) and $D(10,300)\left(A_{\mathrm{V}}=2 \mathrm{mag}\right)$ (see Table 2). For each extinction type (in the MW, LMC or SMC) the charge state distribution $($ cation $=$ red, neutral $=$ black and anion $=$ blue $)$ is shown for the PAHs \#PAH $\left(N_{C}, I P\right)$ : \#1(20,7.6), \#3(40,6.9), of pointgroup $\mathrm{D}_{6 h}$, and, \#5(60,5.25) and \#7(100,4.7) of pointgroup $\mathrm{D}_{2 h}$ (Table 1). $A_{\mathrm{V}}$ is the visual extinction from the edge to the center of the cloud. The change in the fractions translates directly to a change in the observable line strength (for a specific PAH size and PAH abundance). See the main text for a more detailed discussion.

density in the LMC is still only 3/4 of that in the MW (for a diffuse cloud with identical visual extinction, hydrogen density and impinging UV radiation field) assuming that the total PAH column density scales with the carbon abundance and thus metallicity. For the SMC the cation fraction for this PAH is $75 \%$, thus the cationic PAH column density is a factor two lower than in the LMC, and 3/8th that of the MW. In Fig. 6 we show the predicted PAH band strength, i.e., the cation fraction multiplied by metallicity integrated up to a certain $A_{\mathrm{V}}\left(N_{\mathrm{PAH}} \propto A_{\mathrm{V}}\right)$, as a function of that visual extinction $A_{\mathrm{V}}$. The band strength is shown for the diffuse clouds of types B and $\mathrm{C}$ with MW, LMC and SMC metallicities.

The strength of an optical thin absorption line, e.g. a DIB, is directly proportional to the carriers column density (which is proportional to the carbon abundance, the relative PAH abundance and the fraction of cations). In this scenario one would expect DIBs in the LMC and SMC to be about 1.3 and 2.6 times weaker (per $A_{\mathrm{V}}$ ) than in the MW. Stronger DIBs could be expected in the Magellanic Clouds if they emanate from clouds that are exposed to an average interstellar radiation field that is significantly stronger than in the Milky Way; if the total extinction of the cloud is reduced; or if the carbon abundance along a particular line of sight is higher, for example indicated by a higher dust-to-gas ratio. Vice versa, weaker DIBs can be caused by a weak UV field and/or lower metallicity, and thus lower PAH cation abundance. A stronger UV field, $I_{\mathrm{UV}}=10$, would result in a high cation fraction of 75 to $100 \%$, subsequently resulting in SMC DIB strengths half those for the MW, and LMC DIB strengths similar (or even slightly enhanced) to those observed in the MW, despite the lower metallicity of the ISM. Thus, if DIBs were detected in similar (regarding metallicity, density and extinction) interstellar environments, and their 


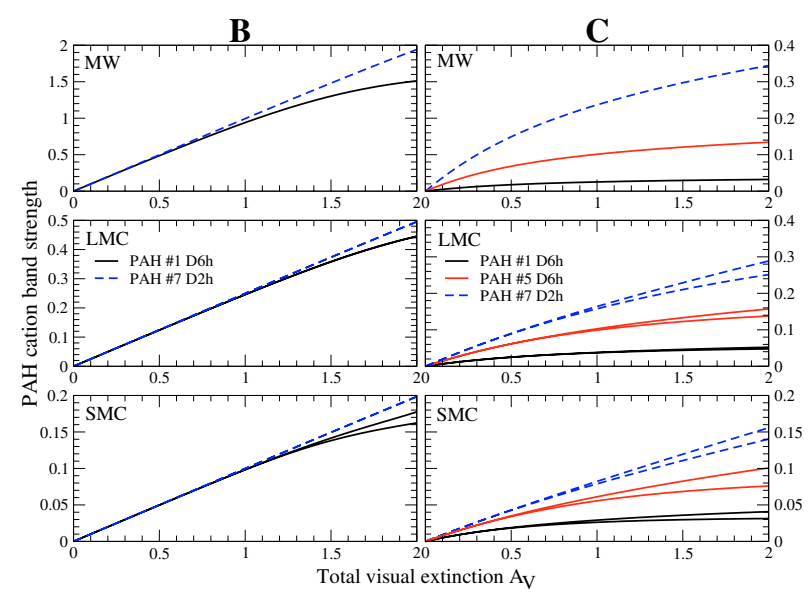

Fig. 6. PAH cation band strength, i.e., the cation fraction multiplied by metallicity integrated up to a certain $A_{\mathrm{V}}$ as a function of that visual extinction $A_{\mathrm{V}}$, for PAHs \#1 $\left(\mathrm{D}_{6 h}\right), \# 5\left(\mathrm{D}_{6 h}\right)$ and \#7 $\left(\mathrm{D}_{2 h}\right)$ of a diffuse cloud of types $B(10,100)$ and $C(1,300)$, for the environments of MW $\left(Z=1.0 Z_{\odot}\right)$, LMC and SMC. It is assumed that $N_{\mathrm{PAH}} \propto A_{\mathrm{V}}$.

carriers would be a PAH-like molecule, then their ratio is a measure of the relative strengths of the effective UV field penetrating the clouds.

Recent studies by Cox et al. (2006a,b) and Welty et al. (2006) show that indeed there are environments within the LMC and SMC where DIBs have strengths similar to those observed for Galactic UV dominated diffuse clouds, as well as those where DIBs are very weak or even absent, typical for shielded Galactic diffuse clouds. It is therefore important to obtain information on the strength of the UV field permeating the Magellanic Clouds. $\mathrm{H}_{2}$ excitation can be used to get a handle on the $I_{\mathrm{UV}} / n_{\mathrm{H}}$ ratio (Black \& van Dishoeck 1987). Also, if present, $C_{2}$ rotational lines can be used to independently derive $n_{\mathrm{H}}$ (Van Dishoeck \& Black 1989). Possibly, more stringent results could be obtained from detailed modelling of specific Magellanic Cloud diffuse lines of sight. However, currently, the information available is not sufficient to adequately constrain the hydrogen density and the temperature structure. Additionally, reality is more complex, and most sightlines probe a complex of individual or connected interstellar clouds.

In the models presented above we refrained from introducing additional PAH destruction and formation processes, such as (de)hydrogenation, fractionation, $\mathrm{C}_{2} \mathrm{H}_{2}$ and $\mathrm{C}_{n}$ photodissociation and $\mathrm{C}^{+}$addition. These processes are not well understood for the large carbon molecules, such as PAHs and fullerenes, considered here. Destruction of carbonaceous molecules by removal of carbon is likely to be important only for small, i.e. less than 20 carbon atoms, molecules. For large PAHs, more than 30 carbon atoms, the effective photo-dissociation drops rapidly with size (Allain et al. 1996a,b; Le Page et al. 2001, 2003). Furthermore, the photo-ionization rate is competing with the bond destruction rate for suitable photons. Predictions by Le Page et al. (2001) suggest that $\mathrm{C}_{2} \mathrm{H}_{2}$ photodissociation rates rapidly drop with increasing $\mathrm{PAH}$ size, for hydrogenated PAHs between 20 to 100 carbon atoms exposed to a standard interstellar radiation field and typical diffuse cloud conditions. For dehydrogenated PAHs (which may occur for strong UV fields) the photo-dissociation is more efficient and $\mathrm{C}_{2} \mathrm{H}_{2}$ loss may also become important for larger $\left(N_{\mathrm{C}}>\right.$ 30) PAHs. Allain et al. $(1996 \mathrm{a}, \mathrm{b})$ predict that most PAHs with less than 50 carbon atoms are destroyed through $\mathrm{C}_{2} \mathrm{H}_{2}$ ejection which only requires about $5.5 \mathrm{eV}$. Note that this is more than is required for $\mathrm{H}$ and $\mathrm{H}_{2}$ loss $(\sim 4-5 \mathrm{eV})$ which is the preferred photo-dissociation process. In addition, $\mathrm{C}_{2} \mathrm{H}_{2}$ loss is higher for catacondensed PAHs than for the peri-condensed PAHs, i.e. UV photons convert open structures to compact structures. Loss of a $\mathrm{C}, \mathrm{C}_{2}$ or $\mathrm{C}_{3}$ requires activation energies of about 7 to $8 \mathrm{eV}$ (Leger et al. 1989), which is significantly higher than the energy required for $\mathrm{H}$ loss (about $5 \mathrm{eV}$ ). Large PAHs $\left(N_{\mathrm{C}}>50\right)$ are photostable as they can redistribute photon energies efficiently, and can remain in their normal hydrogenation state even for radiation fields of $I_{\mathrm{UV}}=100$ (Le Page et al. 2001). PAH anions are more sensitive to loss/abstraction of small hydrocarbons than PAH neutrals or cations. If sufficiently present $\mathrm{C}^{+}$addition can (partly) balance the carbon photo-dissociation processes. Two photon PAH destruction processes are considered to contribute significantly for radiation fields $I_{\mathrm{UV}}>10^{3}$. Again, experimental data for these processes is only available for smaller (up to 20 carbon atoms) PAHs. To understand the importance of photo-dissociation of carbon atoms from large PAHs and fullerenes there is a great need for theoretical calculations and/or experimental laboratory data.

Madden et al. (2005) found that for dwarf galaxies the $\mathrm{PAH}$ band strength (normalized to the number of very small grains) is proportional to their metallicity, possibly because of the hardness of the radiation field, or alternatively the lack of PAH producing AGB stars. Vermeij et al. (2002) studied the infra-red PAH emission from H II regions in the LMC and SMC and inferred that PAHs with an open uneven structure are dominant in Galactic and non-30 Dor LMC H II regions, while compact PAHs are dominant in 30 Dor and SMC H II regions, in line with a strong UV field impinging these regions. Obviously, the environments probed by these studies differ in many ways (extinction, density, UV field strength) from the diffuse medium studied in this work, and one should take caution when comparing.

\subsection{Case study: SMC bar (AzV 18) versus SMC wing (AzV 456) sightlines}

Within the SMC there are two distinct types of diffuse sightline. The lines of sight towards the SMC bar (e.g. AzV 18) show no UV bump, a strong far UV extinction, and no DIBs, whereas, the (only) line of sight towards the SMC wing ( $\mathrm{AzV}$ 456) shows the UV bump, a flatter MW like far-UV extinction and DIBs strengths similar to those observed in the Galactic $\zeta$-type diffuse cloud toward HD 149757. Dust extinction properties are well known for lines of sight towards the early type SMC stars $\mathrm{AzV} 456$ and $\mathrm{AzV} 18$ (Gordon et al. 2003; Cartledge et al. 2005). Both sightlines have a similar visual extinction $A_{\mathrm{V}}$ of 0.57 and $0.49 \mathrm{mag}$, respectively (Table 3 ). The line of sight towards $\mathrm{AzV} 18$ has a gas-to-dust ratio about 3.5 times that towards $\mathrm{AzV} 456 ; 19.3 \times 10^{21}$ and $5.6 \times 10^{21} \mathrm{~cm}^{-2} \mathrm{mag}^{-1}$, respectively. Additionally, they differ in the observed molecular hydrogen fraction $f$ as well as the $\mathrm{H}_{2}$ excitation temperature: $f_{\mathrm{H}_{2}}=0.045$ and 0.525 , and $T_{\mathrm{ex}}=82 \pm 175 \mathrm{~K}$ and $270 \pm 52 \mathrm{~K}$ for AzV 18 and $\mathrm{AzV}$ 456, respectively (Cartledge et al. 2005). The $\mathrm{H}_{2}$ formation rate is proportional to $n_{\text {dust }} \times n_{\mathrm{H}} \times \sqrt{T}$, with $n_{\text {dust }}=x_{\text {dust }} n_{\mathrm{H}}$. The $\mathrm{H}_{2}$ abundance is thus determined by the dust abundance and the ratio $I_{\mathrm{UV}} / n_{\mathrm{H}}$. Hence, it seems plausible that the high $\mathrm{H}_{2}$ fraction toward $\mathrm{AzV} 456$ compared to $\mathrm{AzV} 18$ is a direct consequence of the larger dust-to-gas ratio and a lower $I_{\mathrm{UV}}$ toward the former.

If the metallicity $Z$ increases (as indicated by the dust-to-gas ratio), then the cation fraction decreases, resulting in a predicted DIB strength ratio between $\mathrm{AzV} 456$ and $\mathrm{AzV} 18$ of about 3 
Table 3. The reddening, ratio of the total-to-selective visual extinction, visual extinction, atomic gas-to-dust ratio, molecular gas-to-dust ratio and the total hydrogen gas-to-dust ratio are given for the diffuse interstellar SMC clouds in the lines of sight towards the bar star AzV 18 and the wing star AzV 456.

\begin{tabular}{llll}
\hline \hline Parameter & AzV 456 & AzV 18 & Ref \\
\hline$E_{B-V}$ & $0.30 \pm 0.03$ & $0.17 \pm 0.01$ & 1,2 \\
$R_{\mathrm{V}}$ & $2.19 \pm 0.2$ & $2.90 \pm 0.4$ & 1 \\
$A_{\mathrm{V}}$ & $0.57 \pm 0.08$ & $0.49 \pm 0.11$ & 1 \\
$N(\mathrm{H} \mathrm{I}) / A_{\mathrm{V}}\left(\times 10^{21}\right)$ & 2.6 & 18.4 & 1 \\
$N\left(\mathrm{H}_{2}\right) / A_{\mathrm{V}}\left(\times 10^{21}\right)$ & 1.49 & 0.47 & 1 \\
$N\left(\mathrm{H}_{\mathrm{tot}}\right) / A_{\mathrm{V}}\left(\times 10^{21}\right)$ & 5.6 & 19.3 & 1 \\
$f_{\mathrm{H}_{2}}$ & 0.525 & 0.045 & 1 \\
\hline
\end{tabular}

(1) Cartledge et al. (2005); (2) Cox et al. (2006b).

for a cationic PAH carrier, all other physical parameters being the same. The ratio for a specific DIB in these two SMC lines of sight may be further influenced most significantly by the ambient $I_{\mathrm{UV}}$ because the differences in temperature and hydrogen density are small.

Note then that DIBs of almost Galactic strength have been observed along the line of sight towards $\mathrm{AzV}$ 456, while not towards $\mathrm{AzV}$ 18. If the DIBs towards $\mathrm{AzV} 18$ would indeed be at least a factor of three weaker with respect to those toward $\mathrm{AzV} 456$ then they could have remained undetected up to now, as current upper limits on equivalent widths are only half those of DIBs observed for the Galactic $\zeta$-type sightline toward HD 149757 (Cox et al. 2006b). If upper limits would be observed below the level of the above predicted strength this would hint strongly towards unaccounted for destruction processes. At the moment, the absence of DIBs can be explained satisfactorily by the increased ionization level together with a decrease in carbon abundance, relative to the SMC-wing sightline. For AzV 456 the $2175 \AA$ bump, often associated with carbonaceous material, is strong, while non-existent for $\mathrm{AzV}$ 18. This lack of carbonaceous material agrees with the higher gas-to-dust ratio, lower dust-to-gas ratio, lower metallicity and thus weak (or absent) DIBs observed for $\mathrm{AzV}$ 18. In addition, a cosmic mixture of PAHs (and other complex carbonaceous molecules) may partly contribute to this $2175 \AA$ UV extinction feature (e.g. Lee \& Wdowiak 1993; Boulanger et al. 1994; and Li \& Draine 2001). Lack of PAH-like bump carrier molecules may thus be directly related to a reduced presence of molecules that can give rise (e.g. electronic transitions of the cation can occur in the visible) to the diffuse interstellar bands. This is consistent with our results.

Li \& Draine (2002) studied the molecular cloud SMC B1 \# 1. It has a density of $n_{\mathrm{H}}=1000 \mathrm{~cm}^{-2}$, and extinction $A_{\mathrm{V}}=2.0 \mathrm{mag}$, with an extinction curve of $R_{\mathrm{V}}=4.1$ and metallicity $Z \sim 0.1 Z_{\odot}$ (the gas-to-dust ratio $N(\mathrm{HI}) / A_{\mathrm{V}}=21 \times$ $10^{21}$, similar to $\mathrm{AzV} 18$ ), and found it exposed to an average radiation field of $I_{\mathrm{UV}}=2-3$. In terms of $I_{\mathrm{UV}} / n_{\mathrm{H}}$ this roughly corresponds to our model cloud $C$. If applicable, this would suggest PAH band strengths about twice as strong. Unfortunately, the molecular cloud itself is unsuited for optical observations, and the physical conditions of even nearby diffuse clouds can be vastly different.
Applying diffuse cloud models to specific sightlines requires knowledge of some properties of the gas and dust probed (e.g. (dust) extinction, UV field, metallicity, gas phase abundances of key species, etc.). Once available, this allows for detailed modelling of specific situations. Currently, the models can be used "only" for uncovering general trends in the behaviour of large carbonaceous molecules (such as PAHs) in environments with specific differences.

Acknowledgements. We thank Pascale Ehrenfreund and Lex Kaper for their encouragement and constructive comments, and the referee for several constructive suggestions. N.C. acknowledges support from NOVA.

\section{References}

Allain, T., Leach, S., \& Sedlmayr, E. 1996a, A\&A, 305, 602 Allain, T., Leach, S., \& Sedlmayr, E. 1996b, A\&A, 305, 616 Bakes, E. L. O., \& Tielens, A. G. G. M. 1998, ApJ, 499, 258 Black, J. H., \& van Dishoeck, E. F. 1987, ApJ, 322, 412 Bohlin, R. C., Savage, B. D., \& Drake, J. F. 1978, ApJ, 224, 132 Boulanger, F., Prevot, M. L., \& Gry, C. 1994, A\&A, 284, 956 Cartledge, S. I. B., Clayton, G. C., Gordon, K. D., et al. 2005, ApJ, 630, 355 Cox, N. L. J., Cordiner, M. A., Cami, J., et al. 2006a, A\&A, 447, 991 Cox, N. L. J., Cordiner, M. A., Ehrenfreund, P., et al. 2006b, A\&A, in press Dickey, J. M., \& Garwood, R. W. 1989, ApJ, 341, 201

Ehrenfreund, P., Cami, J., Jiménez-Vicente, J., et al. 2002, ApJ, 576, L117 Fitzpatrick, E. L. 1999, PASP, 111, 63

Fitzpatrick, E. L. 2004, in Astrophysics of Dust, ed. A. N. Witt, G. C. Clayton, \& B. T. Draine, ASP Conf. Ser., 309, 33

Gordon, K. D., Clayton, G. C., Misselt, K. A., Landolt, A. U., \& Wolff, M. J. 2003, ApJ, 594,279

Heger, M. L. 1922, Lick Observatory Bulletin, 337, 141

Herbig, G. H. 1995, ARA\&A, 33, 19

Issa, M. R., MacLaren, I., \& Wolfendale, A. W. 1990, A\&A, 236, 237

Jeyakumar, S., \& Stutzki, J. 2003, in SFChem 2002: Chemistry as a Diagnostic of Star

Formation, proceedings of a conference held August 21-23, 2002 at University of

Waterloo, Waterloo, Ontario, Canada N2L 3G1, ed. C. L. Curry, \& M. Fich (Ottawa, Canada: NRC Press), 309

Kaufman, M. J., Wolfire, M. G., Hollenbach, D. J., \& Luhman, M. L. 1999, ApJ, 527, 795

Le Page, V., Snow, T. P., \& Bierbaum, V. M. 2001, ApJS, 132, 233

Le Page, V., Snow, T. P., \& Bierbaum, V. M. 2003, ApJ, 584, 316

Lee, J.-K., Rolleston, W. R. J., Dufton, P. L., \& Ryans, R. S. I. 2005, A\&A, 429, 1025

Lee, W., \& Wdowiak, T. J. 1993, ApJ, 410, L127

Leger, A., \& D'Hendecourt, L. 1985, A\&A, 146, 81

Leger, A., D’Hendecourt, L., Boissel, P., \& Desert, F. X. 1989, A\&A, 213, 351

Li, A., \& Draine, B. T. 2001, ApJ, 554, 778

Li, A., \& Draine, B. T. 2002, ApJ, 576, 762

Madden, S. C., Galliano, F., Jones, A. P., \& Sauvage, M. 2005, ArXiv Astrophysics e-prints Maloney, P., \& Black, J. H. 1988, ApJ, 325, 389

Maloney, P. R., \& Wolfire, M. G. 1996, in CO: Twenty-Five Years of Millimeter-Wave Spectroscopy, IAU Symp., 170, 299

Misselt, K. A., Clayton, G. C., \& Gordon, K. D. 1999, ApJ, 515, 128

Pak, S., Jaffe, D. T., Van Dishoeck, E. F., Johansson, L. E. B., \& Booth, R. S. 1998, ApJ, 498, 735

Prevot, M. L., Lequeux, J., Prevot, L., Maurice, E., \& Rocca-Volmerange, B. 1984, A\&A, 132,389

Rocca-Volmerange, B., Prevot, L., Prevot-Burnichon, M. L., Ferlet, R., \& Lequeux, J. 1981, A\&A, 99, L5

Rolleston, W. R. J., Trundle, C., \& Dufton, P. L. 2002, A\&A, 396, 53

Ruiterkamp, R., Cox, N. L. J., Spaans, M., et al. 2005, A\&A, 432, 515

Sollerman, J., Cox, N., Mattila, S., et al. 2005, A\&A, 429, 559

Spaans, M. 1996, A\&A, 307, 271

Spaans, M., \& Van Dishoeck, E. F. 1997, A\&A, 323, 953

Stutzki, J. 2001, Ap\&SS, 277, 39

Van der Zwet, G. P., \& Allamandola, L. J. 1985, A\&A, 146, 76

Van Dishoeck, E. F., \& Black, J. H. 1988, ApJ, 334, 771

Van Dishoeck, E. F., \& Black, J. H. 1989, ApJ, 340, 273

Vermeij, R., Peeters, E., Tielens, A. G. G. M., \& Van der Hulst, J. M. 2002, A\&A, 382, 1042

Welty, D. E., Federman, S. R., Gredel, R., Thorburn, J. A., \& Lambert, D. L. 2006, [arXiv: astro-ph/0603332]

Wolfire, M. G., Hollenbach, D., \& Tielens, A. G. G. M. 1989, ApJ, 344, 770

Wolfire, M. G., McKee, C. F., Hollenbach, D., \& Tielens, A. G. G. M. 1995, ApJ, 453, 673 